tors in some unique, recognizable, and durable way; and young people at school, college, or university should be approached to obtain their consent to the use of their organs after death.

Good results from transplantation depend on good tissue matching, and this can be achieved only if there is an adequate supply of donor organs and some registry for potential recipients and donors. Many family doctors would like to be able to tell their patients whom to contact if they wish to donate their organs after their death. This may be a task that could be tackled by one or more voluntary organizations, but it is likely to be expensive, and experience in Europe ${ }^{4}$ suggests that a computer is essential if the system is to be efficient. Can a voluntary organization provide a complex service of this kind ? It would seem to be a task more suitably undertaken by the Department of Health and Social Security. But on the question of individual consent the Government is right to postpone changes in the law until public opinion is clearly in their favour.

\section{Arteriography in Splenic and Hepatic Disease}

Selective arteriography of the coeliac axis and its branches is an important technique for investigating diseases of the liver, spleen, and pancreas. The aorta is approached percutaneously from the femoral artery, and contrast medium is injected through the catheter into the orifices of the emergent arteries. ${ }^{1-3}$

The technique is valuable in detecting tumours, because these disturb the normal vascular anatomy of the organ. In liver cancer the normal vessels are displaced and distorted, while abnormal vessels are seen entering the area of the tumour. Another noteworthy feature is the diffuse staining of the tumour due to an accumulation of contrast medium in its capacious, thin-walled, vascular spaces. ${ }^{4}$ Cysts and tumours of the spleen are also well delineated, and an indication of the degree of malignancy of the condition is often provided. ${ }^{5}$ Selective arteriography is useful in the early diagnosis of pancreatic cancer, ${ }^{6}$ in which displacement, stenosis, and obliteration of normal vascular channels are more usually seen than are tumour vessels or tumour staining. This may be due to the necrotic, cystic nature of many pancreatic cancers. $^{7}$ The method has also been used with advantage in delineating the portal vein in those cases of portal hypertension in which the standard splenoportography performed percutaneously through the splenic parenchyma proves unsatisfactory, or when the spleen has already been removed. $^{3} 8$

L. Blendis, L. Kreel, and $R$. Williams have recently analysed a series of 161 selective coeliac angiograms in an attempt to correlate the comparative sizes of the coeliac axis

Ïdman, P., Acta Radiologica, 1956, 45, 1.

- Ödman, P., Acta Radiologica, 1958, Suppl. 159.

- Stulberg, H. J., and Bierman, H. R., Radiology, 1965, 85, 46.

- Rösch, J., Clinical Radiology, 1966, 17, 183.

Lunderquist, A., Acta Radiologica, 1965, Suppl. 235

Sammons, B. R., Neal, M. P., Armstrong, R. H., and Hager, H. G., American fournal of Roentgenology, Radium Therapy, and Nuclear Medicine, 1967, 101, 345.

- Boijsen, E., Ekman, C.-A., and Olin, T., Acta Chirurgica Scandinavica, $1963,126,315$

- Blendis, L., Kreel, L., and Williams, R., Gut, 1969, 10, 85. and the splenic and hepatic arteries with the size of the spleen (measured by angiography) in conditions in which there was splenomegaly and in liver disease. ${ }^{9}$ They also investigated the relationship between splenic blood flow and vascular size. $\underline{T}$ Of their cases half were controls suffering from other abdomi- $\bar{z}$ nal conditions necessitating angiography, and about a third $\stackrel{\otimes}{\varrho}$ of these had systemic hypertension. The remainder consisted $c$ mainly of hepatic cirrhosis (some with portacaval shunts), $\underset{\vec{s}}{\vec{\rho}}$ hepatic tumours, and tropical splenomegaly.

Both the coeliac axis and the splenic artery were dilated in patients with cirrhosis and in those with portacaval $\overline{\bar{D}}$ anastomoses. There was also dilatation of these arteries in $\bar{\phi}$ tropical splenomegaly, and in most cases of portal-vein thrombusis and liver tumour. By contrast the hepatic artery was not dilated in cirrhosis, tropical splenomegaly, or portal- $\overrightarrow{-}$ vein thrombosis, but there was significant widening in cases $\vec{\omega}$ of portacaval anastomosis and liver tumour. There was $\stackrel{\rho}{\circ}$ lengthening of the splenic artery in patients with cirrhosis 3 and in those with a portacaval anastomosis, but not in cases of tropical splenomegaly.

The spleen was enlarged in cases of cirrhosis but was within the normal range in the great majority of patients with a $\vec{N}$ portacaval shunt. There was a close correlation between the width of the splenic artery and the size of the spleen in cirrhotic and control patients, but no such relation existed between spleen size and the length of the splenic artery, or $\vec{c}$ the widths of the hepatic artery and the coeliac axis. There was moderate splenomegaly in patients with liver tumours irrespective of the presence of cirrhosis. The total blood $\mathscr{\odot}$ flow in the spleen was related to the degree of enlargement of the organ. It also correlated with the width of the splenic artery in cases of cirrhosis and blood dyscrasias but not in tropical splenomegaly.

This investigation throws light on the gross vascular $\frac{0}{\infty}$ changes occurring in diseases of the liver and spleen. Of particular interest is the effect of portacaval anastomosis in widening the hepatic artery in cirrhotic patients. The hepatic arterial dilatation associated with liver tumours can be explained as due to an increased demand for arterial blood, but the enlargement of the spleen and the dilatation of the splenic artery require further investigation. The failure of portacaval anastomosis to narrow the dilated splenic artery 3 . in cirrhosis is also surprising in view of the reduction in $\dot{\delta}$ size of the spleen that follows this operation. More work will be necessary to confirm these findings and assess their $\frac{0}{2}$ significance.

\section{Pyrexia of Unknown Origin}

One of the commonest clinical problems a patient may $\stackrel{\mathscr{D}}{\rightarrow}$ present is a continuing pyrexia in the absence of localizing symptoms and signs. A systematic approach to its elucidation includes a careful history, frequent physical examination, $\stackrel{\mathbb{Q}}{\stackrel{\oplus}{Q}}$ four-hourly records of temperature and pulse, and certain $\stackrel{\mathbb{Q}}{\mathscr{Q}}$ investigations. When a specific system is found to be affected, efforts are directed to its investigation and the patient is noo longer regarded as an ambiguous case of P.U.O.

The concept of P.U.O. has changed with the advent of chemotherapy. Inadequate treatment may mask certain

1 Effersøe, P., Danish Medical Bulletin, 1968, 15, 231.
2 Effersøe, P., Danish Medical Bulletin, 1968, 15, 240. 
phenomena, though fever persists; and, conversely, drug fever may itself complicate the picture. The temperature chart repars study, for it may suggest malaria, Hodgkin's disease, typhoid, or relapsing fever. A routine should include examination of a mid-stream specimen of urine, faeces, throat swab, chest radiograph, leucocyte count, stained blood film, and blood culture. Serum for antibody levels is obtained early in the illness as a baseline for comparison with convalescent-phase sera. The cerebrospinal fluid is examined if there is persistent headache or the suspicion of meningism. If a diagnosis cannot be established by this primary investigation, then other possibilities demand consideration-such as a localized collection of pus, a collagen disorder, hepatic cirrhosis, reticulosis, or malignant disease. But despite a systematic approach and exhaustive investigation many pyrexias defy present-day methods of diagnosis, and even follow-up several years later fails to provide a retrospective diagnosis.

P. Effersøe ${ }^{12}$ has conducted such a follow-up of patients who attended the department of contagious diseases at the Blegdamshospital, Copenhagen, during the four calendar years 1960-3 because of continuous fever daily for at least 10 days. During this time 512 periods of continuous fever were documented in 494 patients. The broad diagnostic categories for these bouts of fever comprised infections in $69 \%$, vascular disorders (8\%), neoplasia (6\%), and collagen disorders $(3 \%)$, but no diagnosis could be established in 34 $(7 \%)$ cases. Between the ages of 15 and 65 years the sex incidence was equal, whereas below 15 years there were twice as many boys and over 65 years there were twice as many women as men in all diagnostic categories.

In the spring of 1967 -that is, three to seven years after their period of continuous fever-Effersøe ${ }^{2}$ traced the subsequent health of the 34 patients in whom the fever had remained of unknown origin. The cause of the fever was found to have been established with certainty only in two instances, both within the first year; one patient showed evidence of Hodgkin's disease and the other of the Budd-Chiari syndrome of hepatic-vein thrombosis. In six patients a tentative retrospective follow-up diagnosis of cytomegalovirus infection seemed possible. Follow-up examination underlined the facts that it is only rarely possible to uncover subsequently the cause of continuous fever which has defied initial diagnosis and that the majority of such patients completely recover.

\section{Immigrants and Venereal Disease}

In a report ${ }^{12}$ presented to the executive board of the World Health Organization in 1964 the Director General described movements of population as one of the important factors concerned in the spread of venereal diseases. It is indeed well known that special temptations assail those who are temporarily or permanently separated from their families and homes, whether they are businessmen visiting Continental capitals, commercial travellers, seamen, long-distance lorry drivers, migrant workers, or immigrants acting as forerunners for their wives and families. It was inevitable, therefore, that immigrants to this country, whether temporary or permanent, would help to inflate the numbers of cases of venereal infection.

Over the years the extent of the contribution provided by these newcomers, who seem for the most part to catch these infections here rather than bring them with them, has been studied by the Co-operative Clinical Group of the Medical Society for the Study of the Venereal Diseases and reported in a series of useful publications. The latest of these studies ${ }^{3}$ concern the incidence of early-that is, primary and secondary-syphilis and of gonorrhoea in 1967, and relate the information to the countries of origin of infected patients. Information on 1,341 patients with primary or secondary syphilis was collected from 186 clinics in England, Wales, and Scotland, comprising $93 \%$ of the cases reported from all clinics in England and Wales in that year and $99 \%$ of those reported in Scotland. The male:female ratio was 4.3 : 1 in England and Wales and 3.0:1 in Scotland, as compared with $2 \cdot 8: 1$ and $2 \cdot 5: 1$ respectively for gonorrhoea. The report attributes this disparity to the fact that male homosexuals form a higher proportion of those with early syphilis than of those with gonorrhoea, but it seems likely that early syphilis acquired by seamen abroad also contributed to the difference. The longer incubation period of syphilis makes it less likely that such a condition will have been recognized and treated in a seaman before he reaches a home port.

Of 996 men treated in England and Wales, 607 (61\%) were born in the United Kingdom and 389 (39\%) in other countries. The biggest contribution, $10 \%$ of the total, came from those born in Asia, followed by $9 \%$ from the West Indies and $8 \%$ from European countries excluding the Mediterranean area. In Scotland there were only 84 cases in men, and 61 of the patients were born in the United Kingdom. Of 233 infected women in England and Wales, 204 $(88 \%)$ were born in the United Kingdom, 7 in the West Indies, and 6 in Asia. Patients from other areas were very few indeed. The report makes the interesting point that the incidence of infectious syphilis appeared to be exceptionally low among African and West Indian negroes who came from areas where yaws is endemic. Only 28 cases of infectious syphilis in females were reported from Scotland, and all these patients were born in the United Kingdom.

As regards gonorrhoea, information from the same clinics in England and Wales showed a total of 27,979 cases in men and 10,087 in women. The numbers for Scotland were 2,092 in men and 843 in women. Of men with gonorrhoea in England and Wales $56 \%$ were born in the United Kingdom, $17 \%$ in the West Indies, and $7.5 \%$ in Asia. In Scotland $88 \%$ of the men were born in Great Britain. Of the women treated in England and Wales, $82 \%$ were born in the United Kingdom and in Scotland $96 \%$.

It is clear that immigrants here as everywhere continue to make a contribution to the increasingly serious problem of venereal disease. But there are indications that, as the numbers of unaccompanied immigrant men decrease and those already here are joined by their families, the contribution they make to the total is relatively less. It is of particular interest that the proportion of men from abroad treated for infectious syphilis in England and Wales in 1966, as determined in the equivalent study for that year, was $50 \%$, but in 1967 it had fallen to $39 \%$. The figures for gonorrhoea in males were $45.4 \%$ in 1966 and 43.6 in 1967 . The proportional contri-

1 Chronicle of the World Health Organization, 1964, 18, 451.

- Chronicle of the World Health Organization, 1965, 19, 7.

3 British fournal of Venerea! Diseases, 1968, 44, 307.

4 British Fournal of Venereal Diseases, 1968, 44, 299. 